\title{
Home Health Cloud Service System Based on Internet of Things
}

\author{
Ji Yu \\ North China Electric Power University, 2 Beinong Road, Changping District, Beijing, China \\ 15886157523@163.com
}

Keywords: Health monitoring, Internet of things, Family diagnosis and treatment products

\begin{abstract}
With the improvement of living standards, people pay more and more attention to the health. However, the current situation of medical treatment is difficult and expensive, strongly contradicted with the increasing medical needs of the people. Community medical service stations are rising day by day, whereas fails to play its full role. Based on the Internet of things, home health cloud service system, can be available at any time to the human health index detection, through the network, so that community doctors can master physical information. To avoid the trouble of queuing up to the hospital, it is convenient for patients to see doctors in time, to discover the abnormal trend of body early, to realize the prevention of some diseases, to communicate online with the users from time to time, focusing on the health of the user, making rehabilitation plan, and popularizing general knowledge of medical care. At the same time, the home environment can be monitored and warned to create a healthy and good home environment.
\end{abstract}

\section{Introduction}

\subsection{Research Background}

With the development of information technology, the application of the Internet of things, cloud technology and artificial intelligence in the development of society. It has played a great role in speeding up the pace of urban construction and development, and has made a solution to the living problems of the residents. Contributions make people see the dawn of using the new generation of information technology to solve all kinds of problems.

At present, many countries study how to use the new generation of information technology to better solve the various problems in urban development. [1] The intelligent medical treatment developed in the background of the Internet of things technology has made patients, medical institutions, and medical equipment more effective. Close interaction allows intelligent medical equipment to analyze disease and body data for patients and create a high efficiency, convenience, and intelligence. The medical service system. The Internet of things promotes the design and innovation of the intelligent equipment to make it more perfect and make the general audience. The group can experience high quality medical services at any time.

Traditional medicine is limited by its own technology and other social factors, resulting in low utilization of resources and accidents. Intelligent medical treatment, through technology such as the Internet of things, closely combines the users of the family with the doctors in the community medical stations. The users check up at home on time, and the doctors can find the problems in time, thus solving the difficulties of the patients in seeing a doctor. [2] At home, patients can test all physical indicators and upload relevant data at any time, so that the system can analyze and predict the disease and achieve early detection, early diagnosis and early treatment.

The technology of Internet of things has been fully applied in medical treatment, and it has solved the urgent need of human health. The rapid development of the medical field, through the relevant technology to patients. Intelligent analysis of body indicators, synchronization of medical, equipment data and drug information, instant processing and more. New, and then improve the core service level of the medical platform, as far as possible to avoid medical security risks. [3] 


\subsection{Research Significance}

The occurrence and development of chronic diseases not only seriously affected people's health and quality of life, but also caused a serious impact on medical and health resources. Serious waste, leading to a rapid rise in medical costs, leading to major public health and social problems. Some diseases once terminally ill will not be able to do anything, but if we can find the disease trend early, is completely curable. How to control it effectively. It is urgent to improve the effect of disease management and quality of life of patients with chronic diseases and save medical expenses in the field of medical and health care. A major task to be solved.

The process of deepening the reform of the medical and health system in our country and the promotion of the classified diagnosis and treatment system. In the context of the degree of degree, using the emerging technology-the home health cloud service system based on the internet of things. Solving the problem of seeing a doctor is an effective exploration. [3] The research significance of this study lies in the realization of "taking the patient as the center". "Heart", taking human health as the core and patient's independent health management as the goal, combining medical technology and testing equipment with the Internet of things.

The appearance of personal intelligent medical products is convenient for families, as part of the most potential development potential in the field of medical devices, in recent years, the traditional health management products with deficient function and complex operation have been gradually replaced in recent years. By using intelligent blood pressure meter, intelligent health hand loop, intelligent disease monitor and other products at home, the user can also The generated medical data is transmitted to the medical service center through the network, so that the prevention and treatment of the disease is timely and efficient.

\subsection{Research Status}

The improvement of people's consumption level has also increased the huge demand for health management services. From the state of going to the hospital only after suffering from the original illness, to regular examination and serious prevention, it has also fully stimulated the steady development of the medical and health industry in our country. Judging from the various policies and provisions issued by the state, the health management industry has been highly valued, and the rational optimization of the intelligent medical platform has an important impact on the promotion of national health. China's health management industry has considerable market potential. Through the Internet of things, big data cloud computing and a series of technologies, people can rely on all kinds of health care products to achieve the management of health records, and real-time measurement of physical signs and indicators. Self-diagnose and communicate with doctors via the internet.

Intelligent health care has become a relatively modern field of technological development in developed countries. In particular, the United States has made great efforts to integrate medical information into its own country, especially in the United States. Intelligent medical field has been developed for many years, constantly break through the bottleneck of technology level, and develop and develop constantly to adapt to the national conditions. In addition to the integration of medical resources and information, hardware and software.

\section{Thinking and Methods}

This paper is mainly about the research and implementation of the home health cloud service system based on the Internet of things that is, based on the Internet of things. Basic carrier, take the wisdom medical treatment as the foundation, carries on the research to its application. Mainly through the investigation, the analysis, the contrast. Discuss and realize the result test.

The research method to be adopted in this subject is the logic of "raising problems, analyzing problems and solving problems". Order, combining theoretical analysis with case analysis, quantitative analysis with qualitative analysis, using characteristic ratio. Comparative analysis and other research methods. [2] 
Based on the idea that the theory originates from practice and acts on practice, the research results are analyzed empirically. According to the feedback information evaluation of application effect and feasibility, the research theory and method body are obtained.

The system and the quantitative model are improved and perfected. Specific research ideas such as Fig. 1.
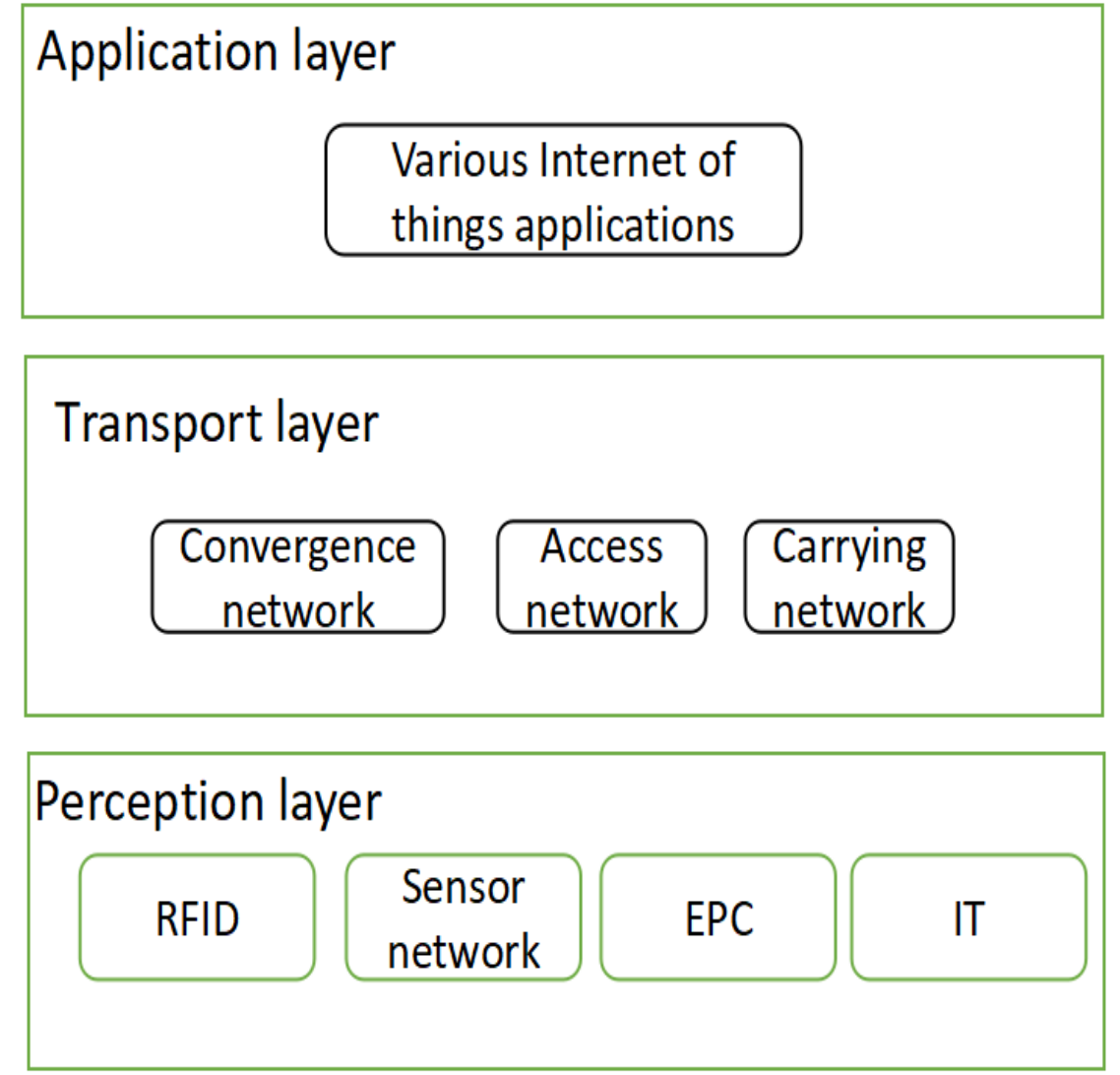

Fig. 1 Research train of thought map

\section{Related Technology Research}

\subsection{Internet of Things Architecture}

In 1999, on the basis of the establishment of Auto-ID technology and Internet of things, the Auto-ID Center of the United States first proposed that "everything can be interconnected through the network", and then clarified the basic meaning of the Internet of things. At present, the more general definition of Inter-net of things is the exchange and communication of information through radio frequency identification (RFID), infrared sensors, global positioning systems, laser scanners, and other information sensing devices that connect any object to the Internet according to an agreed protocol. In order to achieve the intelligent identification, positioning, tracking, monitoring and management of items a network. [3]

With the continuous development of sensor technology, more and more types of sensors for different applications, more and more complete, and more and more intelligent, miniaturized, multi-functional. The important role of new intelligent sensor based on fuzzy theory and intelligent sensor based on neural network technology has been paid attention to. At present, there are various transmission technologies, such as 6Lo WPANM Zig BeetoothUWB, etc. The emergence of new technologies allows the Internet of things to choose appropriate transmission modes for different applications. [4] 


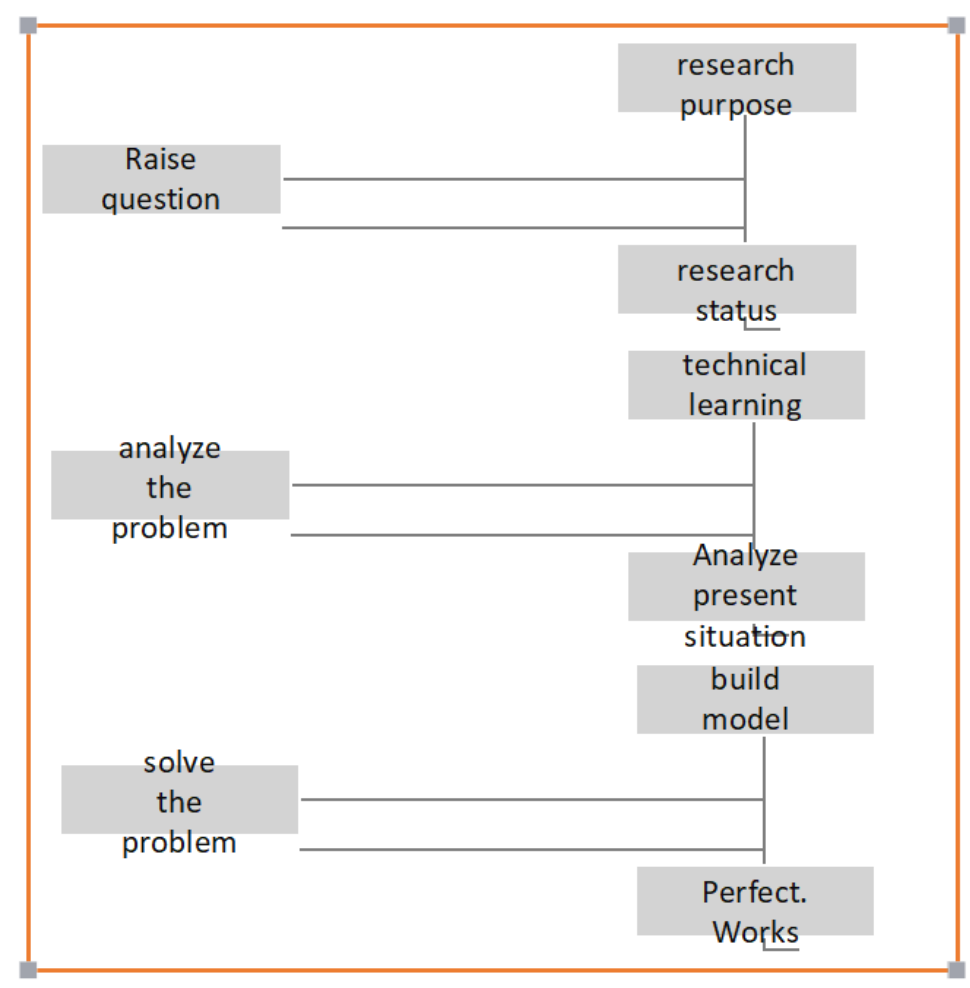

Fig. 2 System architecture

The Internet of things is an important means to realize the intelligent medical treatment and an important tool to carry the construction of the intelligent medical treatment. Therefore, in order to apply the intelligent medical city, we must first study the structure of the Internet of things to ensure the implementation of the intelligent medical application. The generally accepted architecture of the Internet of things is divided into three layers, namely, the perception layer, the transport layer, and the application layer. The system architecture is shown in Fig. 2.

\subsection{Cloud Service System}

NIST defines cloud computing as a ubiquitous, convenient, on-demand, shared, configurable computing resource (such as network, server, storage, etc. A model for network access that enables the rapid supply and release of computing resources through minimal management or interaction with service providers. [5] It distributes computing tasks across a large pool of computers. And support all kinds of application systems in the strong security architecture, the user obtains the sharing information according to the need, the fast computation and the various software services, Networks and application architectures that provide resources are collectively referred to as "clouds". The so-called "cloud" refers to a variety of computing centers distributed in Internet. The cloud contains tens of thousands or even hundreds of thousands of computers or servers.

Cloud computing has five basic features, namely, on-demand self-service, extensive network access, resource pool, fast flexibility, and billing services based on usage. For example, server computing time and network storage without artificial consultation with service providers. [5] Computing power can be accessed via the network and through standard mechanisms, enables a variety of thin (or fat) client platforms to use. The service provider's resources are pooled, and a variety of services are provided to the customer through the multi-tenant model, providing dynamic physical or virtualized resources based on the customer's needs. Storage and network virtualization realize the flexible allocation of resources. The use of resources can be detected, controlled, counted, and provided transparent service usage for customers and service providers.

\subsection{Module technology}

The design monitoring equipment[6] includes fingerprint identification module, detection module, wireless communication module and controller; fingerprint identification module, detection 
module, wireless communication module are respectively connected with controller; The cloud server is provided with a storage module and a data analysis module; a monitoring device, a public platform server and a medical station terminal are respectively connected with the cloud server; the public platform server is connected with an intelligent terminal; This work can make the community medical station always receive the user's environmental parameters and the user's or the user's family's body condition parameter, facilitate the doctor to master the user's body condition, timely reminds the examination and the doctor, realizes the user and the doctor's interaction from time to time; Give full play to the role of community medical station, at the same time to test the home environment, create a healthy, good living environment.

\section{System Architecture}

\subsection{Hardware equipment.}

Table 1 Hardware equipment

\begin{tabular}{|c|c|}
\hline Device name & model \\
\hline Fingerprint identification module & ATK-AS608 \\
\hline Heart rate acquisition module & APDS-9008 \\
\hline Temperature and humidity sensor & DHT11 \\
\hline Temperature collection module & V1.1 \\
\hline Blood pressure monitoring module & SENSOR101 \\
\hline
\end{tabular}

\subsection{Software function}

The transmission system, Wi-Fi [7] is also known as the Wi-Fi module serial port module, belonging to the networking transmission layer, function is the serial port or TTL level to the embedded module with Wi-Fi wireless network communication standard, built-in wireless network protocol IEEE802.11b.g.n protocol stack and TCP/IP protocol stack. Hardware embedded Wi-Fi module of traditional Wi-Fi can be directly used to access the Internet, is to achieve wireless intelligent Home Furnishing, an important part of M2M networking applications. This work uses wifi module to realize real-time data transmission and storage.

Data processing, build cloud platform, build web page. MySQL [8] database is an open source small relational database management system, with high performance, multi-thread, the relational database management system based on the client / server structure is widely used in the small and medium-sized websites on Internet.

Develop an integrated development environment combining php code and mysql database to store and analyze data. The data is only visible to doctors and users themselves. After doctors analyze the data, they can contact users through Official account. Users can also communicate with doctors online at any time to realize medical intelligence.

\section{Summary}

The home health cloud service system based on the Internet of things, with its modern technology, has solved many problems existing in the current medical industry. Intelligent health equipment will also become an essential and important part of the family in the future. With the help of the Internet of things, smart health hardware will become more functional and integrate technology into the service experience of a product. People can solve their own problems through such intelligent products, instead of relying solely on medical institutions, so that the resources of the whole society can be allocated reasonably. The development of the medical Internet of things and the derivative of personal level intelligent health hardware will have more room for improvement in the future. 


\section{References}

[1] Zheng-hui Sun, Chao-min Li, Gaoliang Zhang.Overview of the application of medical devices in the field of mobile medicine [J]. Beijing Food and Medicine Co., Ltd.2016,23(16):4.

[2] Hui Zhao, Shuyu Hu. The era of medical waste in the whole industry chain information management research [J]. Chinese management information networking, 2016,19 (05): 70-71.

Reference to a book:

[3] Zhenzhen Jiang.A study on the strategies of community health management for chronic illness based on the Internet of things [D]. Anhui Medical University, China.2017.

[4] Yu-yang Wang.A study on the Evaluation Model of Medical Internet of things [D]. Huazhong normal University, China.2017.

[5] Lujun Gao. Research and implementation of [D]. China University of Geosciences wisdom medical system (Beijing), 2013.

[6] Qian Chen.Design of Home Intelligent Health Management Terminal based on Internet of things [D]. Shenyang University of Aeronautics and Astronautics (Shenyang University of Aeronautics and Astronautics).

[7] Information on https://baike.so.com/doc/6923076-7145186.html

[8] Information on https://www.php1.cn/detail/php-e5e45318ad.html 